\title{
Reabilitação respiratória: a abordagem holística da pessoa com DPOC
}

Maria Sampaio*

\section{RESUMO}

A reabilitação respiratória assume-se, nos dias de hoje, como um pilar fundamental no tratamento da doença pulmonar obstrutiva crónica (DPOC). Ao adotar uma abordagem holística da pessoa, tendo em conta as várias manifestações sistémicas da doença, consegue melhorar sintomas, exacerbações, capacidade funcional e vertente psicossocial, promovendo uma melhoria global da qualidade de vida.

As evidências atuais apontam para um benefício na introdução deste programa a partir do momento em que a doença é sintomática e causa impacto nas atividades de vida diária. Este quadro corresponde frequentemente a graus de obstrução do fluxo aéreo ainda moderados - grau GOLD 2 - e a reabilitação nesta fase permite uma maior estabilização ou mesmo regressão das manifestações sistémicas da doença. A capacidade de resposta dos serviços hospitalares que oferecem este tipo de programa é, contudo, muito escassa, limitando-se aos doentes em estádios mais severos - GOLD 3 e, sobretudo, GOLD 4. Torna-se, pois, fundamental investir em programas de reabilitação respiratória comunitários, inseridos nos cuidados de saúde primários, de forma a aumentar a acessibilidade e equidade de cuidados a todos os que deles beneficiam.

Palavras-chave: Doença pulmonar obstrutiva crónica; Reabilitação respiratória.

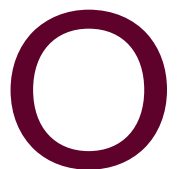
conceito de reabilitação respiratória (RR) nasceu há cerca de duas décadas e tem vindo a ganhar importância pelo progressivo entendimento de que a doença pulmonar obstrutiva crónica (DPOC) é, muito mais do que uma doença pulmonar, uma doença de origem pulmonar com manifestações sistémicas. ${ }^{1}$ Da necessidade de abordar esta patologia em toda a sua complexidade sistémica, surgiu o conceito de RR, definida como uma intervenção global e multidisciplinar baseada na evidência, dirigida a doentes com doença respiratória crónica, sintomáticos e, frequentemente, com redução das suas atividades de vida diária. ${ }^{2}$ A RR pretende, assim, uma abordagem holística e integrada da pessoa com DPOC que tenha em conta as suas várias manifestações físicas e psicossociais. São fortes as evidências que demonstram benefícios no aumento da tolerância ao exercício, na realização das atividades diárias, na autonomia, adesão à terapêutica e qualidade de vida. Por

*USF Garcia de Orta, ACeS Porto Ocidental. outro lado, diminui a dispneia, a ansiedade, o número de exacerbações, de internamentos e o isolamento social. $^{2-3}$

Assim, a RR assume-se hoje como um pilar fundamental do tratamento da DPOC, juntamente com a intervenção farmacológica e a cessação tabágica, sendo um componente insubstituível do tratamento. ${ }^{4}$

Tendo em conta que a DPOC é uma doença com peso relevante, faz sentido que seja um assunto na ordem do dia. De facto, ocupa o $5 .^{\circ}$ lugar como causa de morte a nível mundial com previsões de passar para $3 .^{\circ}$ lugar em 2030. ${ }^{5}$ A nível nacional, os valores de prevalência são discrepantes consoante os estudos. O estudo Pneumobil detetou uma prevalência de 8,96\%. ${ }^{6}$ Já dados publicados em 2013, relativamente a um estudo realizado na região de Lisboa, revelaram uma prevalência de 14,2\% na população com idade superior a 40 anos. ${ }^{7}$ Segundo dados mais recentes do European Health Interview Survey, de 2014, foi encontrada uma prevalência autorreportada de 5,8\%. ${ }^{8}$ Adicionalmente, segundo os últimos dados disponíveis de 2014, a doença é responsável por 
um terço dos internamentos por doença respiratória com custos elevados para o Serviço Nacional de Saúde. ${ }^{9}$ Sabendo desta realidade e do previsível agravamento destes números nas próximas décadas, tendo em conta o envelhecimento da população, é prioritário falar-se em prevenção e adequada gestão da doença. E, portanto, importa à medicina geral e familiar, como faz parte da essência da disciplina, a promoção da prevenção da doença em todas as suas formas. São vários os estudos que demonstram que a RR é uma forma segura, eficaz e barata de prevenção terciária. ${ }^{2}$ Um Programa de RR implica, pois, uma determinada estrutura e uma intervenção multidisciplinar, incluindo médicos (pneumologista e fisiatra), enfermeiros de reabilitação, fisioterapeutas e ainda, idealmente, psicólogos e nutricionistas. A sua estrutura consiste em treino de exercício e das atividades de vida diária, ensino de técnicas de fisioterapia respiratória, educação da pessoa com DPOC de forma a aumentar a capacitação para a autogestão da doença, bem como intervenção nutricional e psicossocial. ${ }^{2,5}$

E é assim, nesta forma de prevenção, que a medicina geral e familiar deve estreitar relações com as especialidades hospitalares, criando pontos de contacto para benefício dos doentes. De facto, os atuais programas de RR são realizados a nível quase exclusivamente hospitalar, nos serviços de fisiatria, o que se traduz numa compreensível insuficiente capacidade de resposta. Na realidade, independentemente da sua capacidade de resposta, a sua existência apenas hospitalar constituiria sempre uma enorme limitação à acessibilidade destes cuidados - porventura não nas grandes cidades, mas na maioria do interior do País, onde o hospital mais próximo se encontra a longa distância. Tal não permite que um doente possa frequentar as sessões de periodicidade bi ou tri-semanal que o programa exige. Acresce o facto, acima referido, de os hospitais não terem efetivamente capacidade para dar resposta a todos os doentes com indicação para RR. Assim, na prática, estes programas restringem-se aos doentes mais críticos e com necessidade de intervenção prioritária, ou seja, doentes em estádios GOLD 3 e, sobretudo, GOLD 4, fundamentalmente referenciados pela pneumologia em contexto de pós-internamento por exacerbação da sua doença. Assim, não é de surpreender que apenas tenham acesso à RR em Portugal menos de
0,5\% dos doentes que dela beneficiariam. ${ }^{9}$ Segundo várias orientações nacionais e internacionais, beneficiam de um programa de RR todos aqueles com DPOC moderada e, sobretudo, sintomática, isto é, já a partir do grau GOLD 2 com pontuação igual ou superior a dois na Escala de dispneia do Medical Research Councilmodificada (mMRC). ${ }^{2,4,10}$ De facto, o estudo funcional respiratório basal isolado não é preditivo da resposta ao programa de reabilitação, pois apesar de os valores respiratórios serem indicadores da gravidade da doença são por si só insuficientes, uma vez que se relacionam pouco com a dispneia e a tolerância ao exercício. ${ }^{2}$ A sintomatologia apresentada e sobretudo o seu impacto na qualidade de vida são os fatores de grande peso nesta seleção da população a intervencionar. ${ }^{2-4}$ Desta forma, seria extremamente relevante que os médicos de família pudessem orientar os seus doentes em fases mais precoces da DPOC, mas que já veem o seu dia-a-dia afetado pela doença, garantindo-lhes uma adequada acessibilidade e articulação com os cuidados de que beneficiam. O que implica pensar em alternativas aos programas hospitalares.

Existem vários estudos a demonstrar não existir diferenças significativas em termos de resultados major entre os programas de RR hospitalares e os de base comunitária, sendo que é fortemente recomendado que todos os doentes com DPOC e indicação para RR a ela tenham acesso, independentemente do local. ${ }^{10} \mathrm{Um}$ outro estudo recente salientou ainda algumas das vantagens destes programas comunitários, nomeadamente a conveniência dos acessos e maior sensação de normalidade por parte dos participantes, com consequente maior grau de satisfação. ${ }^{11} \mathrm{O}$ Canadá e a Austrália são exemplos de países com feedback positivo desta experiência de extensão extra-hospitalar da RR. ${ }^{11-13}$ Torna-se, assim, fundamental seguir estes exemplos e alargar os Programas de RR aos cuidados de saúde primários (CSP) de forma a aumentar a capacidade de resposta a esta população crescente, melhorando a acessibilidade e a equidade de cuidados. Uma forma possível de o fazer seria através das Unidades de Cuidados na Comunidade (UCC), parte integrante de cada Agrupamento de Centros de Saúde (ACeS). A integração de médicos especialistas em medicina física de reabilitação e em pneumologia nesta Unidade, bem como a aposta na formação nesta área, seria obviamente fulcral 
para a estruturação destes programas. Poder-se-ia, assim, desenvolver uma rede integrada de competências complementares entre si inseridas nos CSP, dedicadas às pessoas com multimorbilidade crónica envolvendo vários aparelhos e sistemas, uma vez que as principais componentes da RR são comuns à prevenção e controlo de um vasto leque de doenças crónicas que não apenas as respiratórias. Seria, provavelmente, esta a opção com maior rentabilização de recursos e melhor relação custo-benefício. Para os ACeS com menos recursos poder-se-ia apostar em programas mais simples dedicados apenas ao treino de exercício, que é já um primeiro passo fundamental para quebrar o ciclo vicioso da inatividade.

Em termos das pessoas com DPOC, estes serviços poderiam oferecer programas de RR aos doentes em estádios mais moderados (GOLD 2) seguidos pelos médicos de família, bem como sessões de manutenção para os doentes mais graves que terminaram o programa hospitalar, algo fundamental para garantir os benefícios a longo prazo.

É certo que a criação deste novo serviço a nível dos CSP exige investimento. Implementados com qualidade, sabe-se, contudo, que estes cursam com uma diminuição efetiva dos custos diretos e indiretos da doença através da diminuição do número de exacerbações, do recurso a consultas, da quantidade de medicação prescrita e do número e dias de internamentos das pessoas com DPOC. E, sobretudo, conseguir-se-ia melhorar a sua qualidade de vida. ${ }^{1-4}$

Está, pois, na hora de mudar o paradigma dos programas de reabilitação como exclusivamente hospitalares e encarar como uma prioridade a necessidade de melhorar a capacidade de resposta às pessoas com patologia respiratória crónica.

\section{REFERÊNCIAS BIBLIOGRÁFICAS}

1. Corhay JL, Dang DN, Van Cauwenberge H, Louis R. Pulmonary rehabilitation and COPD: providing patients a good environment for optimizing therapy. Int J Chron Obstruct Pulmon Dis. 2014;9:27-39.

2. Direção-Geral da Saúde. Orientações técnicas sobre reabilitação respiratória na doença pulmonar obstrutiva crónica (DPOC): circular normativa n. ${ }^{\circ}$ 40A/DSPCD, de 27/10/2009. Lisboa: DGS; 2009.

3. Global Initiative for Chronic Obstructive Lung Disease. Global strategy for the diagnosis, management, and prevention of chronic obstructive pulmonary disease. Fontana: GICOLD; 2017.

4. António C, Gonçalves AP, Tavares A. Doença pulmonar obstrutiva cró- nica e exercício físico [Pulmonary obstructive chronic disease and physical exercise]. Rev Port Pneumol. 2010;16(4):649-58. Portuguese

5. World Health Organization. Chronic respiratory diseases: burden of COPD [website]. Geneva: WHO; [2017]. Available from: http://www. who.int/respiratory/copd/burden/en/

6. Cardoso J, Ferreira JR, Almeida J, Santos JM, Rodrigues F, Matos MJ, et al. Doença pulmonar obstrutiva crónica em Portugal: estudo Pneumobil (1995) e estudo de prevalência de 2002 revisitados [Chronic obstructive pulmonary disease in Portugal: Pneumobil (1995) and 2002 prevalence studies revisited]. Rev Port Pneumol. 2013;19(3):88-95. Portuguese

7. Barbara C, Rodrigues F, Dias H, Cardoso J, Almeida J, Matos MJ, et al. Prevalência da doença pulmonar obstrutiva crónica em Lisboa, Portugal: estudo Burden of Obstructive Lung Disease [Chronic obstructive pulmonary disease prevalence in Lisbon, Portugal: the Burden of Obstructive Lung Disease Study]. Rev Port Pneumol. 2013;19(3):96-105. Portuguese

8. OECD, European Union. Asthma and COPD prevalence. In: Health at a glance: Europe 2016 - State of health in the EU cycle. Paris: OECD Publishing; 2016. p. 82-3. ISBN 9789279631429

9. Guimarães M. Doença pulmonar obstrutiva crónica (DPOC). In: Observatório Nacional das Doenças Respiratórias: relatório 2017. Lisboa: Observatório Nacional das Doenças Respiratórias; 2017. p. 70-45.

10. Marciniuk DD, Brooks D, Butcher S, Debigare R, Dechman G, Ford G, et al. Optimizing pulmonary rehabilitation in chronic obstructive pulmonary disease - practical issues: a Canadian Thoracic Society Clinical Practice Guideline. Can Respir J. 2010;17(4):159-68.

11. McNamara RJ, McKeough ZJ, Mo LR, Dallimore JT, Dennis SM. Community-based exercise training for people with chronic respiratory and chronic cardiac disease: a mixed-methods evaluation. Int J Chron Obstruct Pulmon Dis. 2016;11:2839-50.

12. Cecins $\mathrm{N}$, Landers $\mathrm{H}$, Jenkins $\mathrm{S}$. Community-based pulmonary rehabilitation in a non-healthcare facility is feasible and effective. Chron Respir Dis. 2017;14(1):3-10.

13. Desveaux L, Beauchamp MK, Lee A, Ivers N, Goldstein R, Brooks D. Effects of a community-based, post rehabilitation exercise program in COPD: protocol for a randomized controlled trial with embedded process evaluation. JMIR Res Protoc. 2016;5(2):e63.

\section{CONFLITO DE INTERESSES}

A autora declara não ter quaisquer conflitos de interesse.

\section{FINANCIAMENTO}

O presente trabalho não foi objeto de qualquer tipo de financiamento externo.

\section{ENDEREÇO PARA CORRESPONDÊNCIA}

Maria Sampaio

E-mail: sampmaria@gmail.com

http://orcid.org/0000-0002-8739-6604

\section{Recebido em 15-02-2017}

Aceite para publicação em 08-10-2018 


\section{ABSTRACT}

\section{PULMONARY REHABILITATION: THE HOLISTIC APPROACH OF THE PERSON WITH COPD}

Pulmonary rehabilitation is recognized today as a fundamental cornerstone in the treatment of chronic obstructive pulmonary disease (COPD). By adopting a holistic approach to the patient, taking into account the various systemic manifestations of the disease, it manages to improve symptoms, exacerbations, functional capacity with psychosocial aspects, thereby promoting an overall improvement in quality of life.

Current evidence shows benefit in the implementation of this program from the moment the disease is symptomatic and impacts daily living activities. That often corresponds to moderate stages of airflow obstruction - GOLD 2 - and rehabilitation in this stage allows for a greater stabilisation, or even regression of the systemic manifestations of the disease. The responsiveness of hospital services offering this type of program is, however, very scarce, limited to the patients in more severe stages - GOLD 3, and mainly GOLD 4. Therefore, it is essential to develop community-based pulmonary rehabilitation programs within primary health care, in order to increase availability and equity of care to those who benefit from them.

Keywords: Chronic obstructive pulmonary disease; Pulmonary rehabilitation. 